\title{
Effect of donor arm washing on microbial growth at phlebotomy site in first time blood donors
}

\begin{abstract}
Background: Microbes at the phlebotomy site are the important source of bacterial contamination of blood products. Various methods to reduce their load at phlebotomy site have been tried and have always being improvised upon. Few of the blood centres have adopted a policy of making the donors wash their arms with soap and water before disinfection for phlebotomy. However the utility of this policy has not been studied.
\end{abstract}

Aim: The aim was to study if washing the phlebotomy site with soap and water before blood donation would make an impact or change the microbiota at the site.

Materials and methods: The study included 200 whole-blood donors who were randomly chosen and after obtaining an informed consent were included in the study. The donors were alternately allocated into donation arm washing $(n=100)$ or non washing $(100)$ group. The swabs were taken from the phlebotomy site from both the groups and cultured for microbial growth. The results were compared to see for difference between the groups. The statistical analysis was done using IBM SPSS Statistics Desktop Software version 22.0.

Results: Multiple organisms were isolated in only three of the 40 donors in the arm washed group compared to 24 of the 50 donors evaluated in the control arm and this difference was statistically significant $(\mathrm{p}<0.01)$

Conclusion: This simple add on step of washing the donor arm before donation reduced the microbial floral load at the phlebotomy site.

Keywords: donor arm washing, phlebotomy, SPSS statistics
Volume 6 Issue 6 - 2018

\author{
Harshavardhana, ${ }^{2}$ Abhishekh \\ Basavarajegowda,' Deepa S Anand ${ }^{2}$ \\ 'Jawaharlal Institute of Postgraduate Medical Education and \\ Research, India \\ ${ }^{2}$ Kidwai memorial institute of Oncology, India
}

\begin{abstract}
Correspondence: Abhishekh B,Assistant Professor, Department of Transfusion Medicine, Room no 5042, Superspeciality Block, India, Tel 900825588 ,

Email dradhigowda@gmail.com
\end{abstract}

Received: September 29, 2018 | Published: November 08, 2018

\section{Introduction}

Blood transfusion has been evolving and has come a long way from washed reusable bottles to closed system of Blood collection by single use disposable plastic blood bags. The risk of Transfusion Transmitted Infections has been reduced largely with the advent of improved technologies for testing blood for various infectious agents as well as the improvements in the selection of blood donors itself. Bacterial contamination of blood products especially the platelets is much more common when compared to the incidence of all the transfusion transmitted infections put together viz. the ones regularly screened for like HIV, HBV, HCV, Syphilis and Malaria. ${ }^{1}$ The main source of this bacterial contamination of blood components is the microbiota residing on the donor skin at the site of venipuncture. These are thought to gain entry into the blood bag through the needle. ${ }^{2,3}$ Numerous techniques have been tried to reduce contamination of blood/blood products like diversion pouch, dual step disinfection at the venipuncture site etc. However the risk still persists. ${ }^{4}$

Hand washing has been shown to be very effective in reducing the spread of infections in the hospital as seen in the reduction of hospital acquired infections after improved hand washing techniques among healthcare personnel. ${ }^{5}$ Washing also has shown to reduce the magnitude of microbial flora on the skin. ${ }^{5}$ Some of the blood collection centers implement or ask the donors to wash their arms before donating blood. However the impact of the same has not been studied or reported previously. ${ }^{6}$ This study was undertaken to study if washing the phlebotomy site with soap and water before blood donation would make an impact or change the microbiota at the site.

\section{Materials and methods}

\section{Collection, processing of samples and bacterial identification}

The study included 200 whole-blood donors after being declared fit for donating blood by the doctor from the Blood Center. Participants were randomly chosen and after obtaining an informed consent were included in the study. The donors were alternately allocated into donation arm washing $(\mathrm{n}=100)$ or non washing (100) group. The donors who came under the hand washing group were asked to wash their venipuncture (antecubital) area with free flowing water from the tap in phlebotomy room. Hand washing was clearly instructed as well as demonstrated whenever required. For each donor, in both groups, a two-stage technique that uses antibacterial $10 \%$ povidoneiodine $(10 \% \mathrm{P}-\mathrm{I})$ followed by alcoholic $10 \% \mathrm{P}-\mathrm{I}$ was used. The procedure is summarized as follows: in the first step, swab soaked in antibacterial $10 \% \mathrm{P}-\mathrm{I}$ was applied in ascending and descending pattern for 30 seconds. After an interval of 30 seconds, the second stage, alcoholic $10 \% \mathrm{P}-\mathrm{I}$ was applied in a single movement from the centre outward (whirling pattern). After a subsequent waiting period of 30 seconds, alcohol was allowed to evaporate and dry, following which phlebotomy was performed.

To enumerate and identify bacteria present at the venipuncture site (antecubital fossa) post-disinfection, a sterile cotton wool swab moistened using sterile distilled water for injection was rolled over the phlebotomy site 3 times and transported in a sterile dry tube to the Microbiology laboratory for culture. 
Each swab was plated onto a blood agar plate and incubated for $24 \mathrm{hrs}$ in $5 \% \mathrm{CO}_{2}$. The following day the plates were examined for growth. ${ }^{7}$ Colonies on the plate were examined by Gram stained smears and identified using standard microbiological procedures (Figure 1).

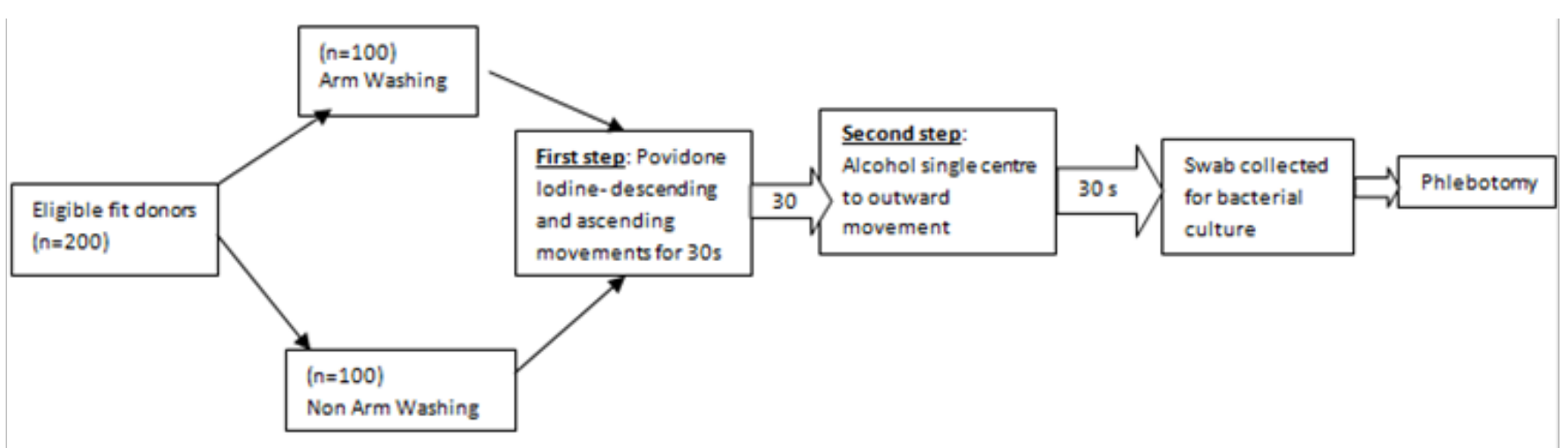

Figure I Processing of samples and bacterial identification.

\section{Donor characteristics}

Characteristics of the participant donor subjects such as age, nature of work, gender, previous donations, last donation interval etc. were collected from the donor records, available in the blood centre, which has information on all blood donation candidates. These data were collected and analyzed for any relationship between donor characteristics and microbial load.

\section{Statistical analysis}

All the data were entered in the Microsoft Excel sheet and the statistical analysis was carried out using IBM SPSS Statistics for Windows, Version 21.0., 2013 (Armonk, NY, IBM Corp.). All the categorical variables were expressed as proportions. Any difference in the percentage of Microbial growth in both intervention and control arms was compared using Chi-square test.

The study was approved by the institute ethics committee.

\section{Results}

The study included a total of 200 donors. 100 each were randomized to either arm washing group and non arm washing group wherein they were either made to wash their arms at the phlebotomy site using soap and water or not. The participants were comparable between groups with respect to their biophysical characters including age, gender, weight, occupation, number of previous donations and time since last donation with no significant difference between the groups in all the categories ( $p>0.05$, Table 1$)$.

Table I Comparison of Biophysical characters of the participants in the study groups.

\begin{tabular}{|c|c|c|c|}
\hline Character & Arm washed $(n=100)$ & Arm not Washed $(n=100)$ & p value \\
\hline \multicolumn{4}{|l|}{ Age(in years) } \\
\hline $18-30$ & 65 & 71 & \multirow{3}{*}{0.65} \\
\hline $31-45$ & 33 & 27 & \\
\hline $46-54$ & 2 & 2 & \\
\hline \multicolumn{4}{|l|}{ Occupation } \\
\hline Students & 28 & 35 & \multirow{5}{*}{0.44} \\
\hline Professionals & 8 & 10 & \\
\hline Clerical work & 41 & 36 & \\
\hline Technical work & 6 & 9 & \\
\hline Miscellaneous & 17 & 10 & \\
\hline \multicolumn{4}{|l|}{ Gender } \\
\hline Male & 96 & 89 & \multirow{2}{*}{0.06} \\
\hline Female & 4 & 11 & \\
\hline \multicolumn{4}{|c|}{ Previous donations (Number) } \\
\hline First time & 40 & 44 & \multirow{4}{*}{0.89} \\
\hline$<5$ & 49 & 45 & \\
\hline 09-May & 9 & 8 & \\
\hline 10 or more & 2 & 3 & \\
\hline
\end{tabular}




\begin{tabular}{|c|c|c|c|}
\hline Character & Arm washed $(n=100)$ & Arm not Washed $(n=100)$ & p value \\
\hline \multicolumn{4}{|c|}{ Time since last donation } \\
\hline First time & 40 & 44 & \multirow{4}{*}{0.06} \\
\hline$<6$ months & 6 & 13 & \\
\hline 6 month-I year & 44 & 28 & \\
\hline More than I year & 10 & 15 & \\
\hline \multicolumn{4}{|l|}{ Weight (In kg) } \\
\hline $50-60$ & 17 & 22 & \multirow{3}{*}{0.52} \\
\hline $61-80$ & 62 & 62 & \\
\hline More than 80 & 21 & 16 & \\
\hline
\end{tabular}

The total number of positive cultures in the arm washed group was lesser $(n=40)$ than that in the control (arm not washed) group $(n=50)$ although the difference was not statistically significant $(\mathrm{p}=0.155$; table 2). The yield of microbial growth varied between the groups : multiple organisms were isolated in only three of the 40 donors in the arm washed group compared to 24 of the 50 donors evaluated in the control arm and this difference was statistically significant $(\mathrm{p}<0.01$; Table 2).

Table 2 Comparison of bacterial growth in study groups.

\begin{tabular}{llll}
\hline Type of Growth & Arm washed $(\mathbf{n}=100)$ & Arm not Washed $(\mathbf{n}=1 \mathbf{0 0})$ & p value \\
\hline Single organism (Gram Positive Bacilli) & 31 & 24 & 0.268 \\
Single organism (Gram Positive Cocci) & 6 & 2 & 0.149 \\
Multiple organisms & 3 & 24 & 0.0001 \\
No Growth & 60 & 50 & 0.155 \\
Total & 40 & 50 & \\
\hline
\end{tabular}

\section{Discussion}

The study was conducted by randomizing 100 people each into two groups which were instructed to wash their arm (phlebotomy site) with soap and water and the other group which did not receive any such instructions before blood donation. The Biophysical characteristics of the donors in both the groups were comparable Majority of the donors (up to $2 / 3^{\text {rd }}$ ) were in the age group of 18 to 30 years. ${ }^{8}$ This is the major age group to which donors belong to in most of the blood banks in the country. The numbers of females in each group were also comparable with no statistically significant difference between groups $(\mathrm{p}=0.06)$ which is around $10 \%$ in our national average as well as Global database of WHO. Students constituted around $1 / 3^{\text {rd }}$ of the donors. The donors in both groups were also comparable with respect to number of donations and time since last donation. The number of donations and time since last donations may have an effect on the microbiota at the phlebotomy site as repeated phlebotomy may have changed the skin contour and the duration since last donation is important for the healing and remodeling of the previous phlebotomy scar. ${ }^{9}$

The percentage of donors which yielded growth in the unwashed arm was in $50 \%$ compared to $40 \%$ in the washed arm. So there was a difference of $10 \%$ amongst the groups. Though this difference was not statistically significant, it was notable that multiple organisms were isolated in $24 \%$ of the donors in control arm whereas only $3 \%$ in the intervention arm yielded multiple organisms $(\mathrm{p}<0.01)$. The majority of the growth isolated from the intervention arm was single type of organisms.

Bacterial contamination of platelets continues to be a problem for transfusion. Major source of these contaminations is the microbial flora on the skin at phlebotomy site. The microbial flora at the skin site could be either transient or resident. Resident flora is the organisms that are constantly present and cannot be removed permanently. They prevent permanent colonization of the body by other organisms. On the other hand, transient flora, as the name suggests, are present at some point of time, but not always. They may be potentially pathogenic or non-pathogenic too. It is this transient flora which is expected to be removed by washing with soap and water.

In present study the organisms isolated were Gram positive Bacilli mainly diptheroids and Bacillus which are usually nonpathogenic, Gram positive Cocci include Staphylococci, Streptococci and Enterococci which can be pathogenic. However, some Gram positive cocci like Micrococci are non-pathogenic.

The human skin is constantly \& continuously bombarded by organisms present in the environment. Depending on the individual's personal hygiene, it can also be contaminated by one's own secretions $\&$ excretions. The area of the body, the clothing worn, his/her occupation and even the environment have a bearing on the type \& nature of flora.

Cultures from the skin have frequently shown diphtheroids, Staphylococci (aerobic and anaerobic) and Gram-positive aerobic, spore-bearing bacilli. Also seen on the skin are Streptococcus viridans, Streptococcus fecalis, and Gram-negative bacilli like E.coli, Proteus and other intestinal organisms and even non-pathogenic mycobacteria. Candida albicans and Cryptococci are also seen. ${ }^{10}$

Sometimes, the skin of the face, neck, hands and buttocks carries pathogenic hemolytic Streptococci and Staphylococci. In the case of 
individuals working in hospitals, penicillin-resistant staphylococci may also be seen.

Though the usage of blood bags with diversion pouch have shown to reduce the risk of bacterial contamination, ${ }^{4}$ these bags are a bit costlier when compared to the regular bags and hence many centers in our country may not be readily able to use them. Even centers which are able to use them can still adopt this simple add on step of washing the donor arm before donation in order to reduce the risk of contamination to much lower levels. This does not add inconvenience or cause risk to the donor in any way.

The limitation of the study is that we could not look into whether the presence of the bacteria on the skin actually transforms into contamination of the product. That can only be addressed with a very large study as the contamination of the product has been shown to be less than $1 \%$ in most of the studies. ${ }^{11}$ Studies may be planned in those terms especially a multicentre study to address this issue.

\section{Acknowledgements}

Dr. Prathiba, medical officer at KMIO, Bangalore for recruiting donors for the study.

\section{Conflicting interest}

Authors declare that there is no conflict of interest.

\section{References}

1. Blajchman MA. Incidence and significance of the bacterial contamination of blood components. Dev Biol (Basel). 2002;108:59-67.
2. Puckett A, Davison G, Entwistle CC, et al. Post transfusion septicemia 1980-1989: importance of donor arm cleansing. J Clin Pathol. 1992;45(2):155-157.

3. Cunha G, Leão L, Pimenta F. Bacterial contamination of random-donor platelets in a university hospital in the Midwestern region of Brazil. Transfusion. 2008;48(2):282-285.

4. De Korte D, Curvers J, De Kort WL, et al. Effects of skin disinfection method, deviation bag, and bacterial screening on clinical safety of platelet transfusions in the Netherlands. Transfusion. 2006;46(3):476-485.

5. Larson E. A Causal Link between Hand washing and Risk of Infection? Examination of the Evidence. Infect Control. 1988;9(1):28-36.

6. Patel TG, Shukla RV, Gupte SC. Impact of Donor Arm Cleaning with Different Aseptic Solutions for Prevention of Contamination in Blood Bags. Indian J Hematol Blood Transfus. 2013;29(1):17-20.

7. Gary WP, Elmer WK. Introduction to Microbiology Part-II: Guidelines for the Collection, Transport, Processing, Analysis \& Reporting of Cultures from Specific Specimen Sources. Koneman's Color Atlas and Textbook of Diagnostic Microbiology. 7th ed. 2016:66-110.

8. Piyush Patel, Sangeeta Patel, Manoj Patel. Study of Blood Donors Characteristics at Teaching Hospital, Western India. Natl J Integr Res Med. 2015;6(4):56-62.

9. Perez $\mathrm{P}$, Bruneau $\mathrm{C}$, Chassaigne $\mathrm{M}$, et al. Multivariate analysis of determinants of bacterial contamination of whole blood donations. Vox Sang. 2002;82(2):55-60.

10. Ananathanarayan R, Jayaram P. Normal microbial flora of the human body. Textbook of Microbiology. 9th ed. 2014:621-624.

11. Pietersz RN, Reesink HW, Panzer S, et al. Bacterial contamination in platelet concentrates. Vox Sang. 2014;106(3):256-283. 